\title{
LA UTILIDAD DE LA BIOIMPEDANCIA EN PERSONAS MAYORES
}

\author{
Marina Morela Hernández Patrocinio \\ Enfermera del complejo asistencial de Zamora .Servicio de endocrino. \\ morelatoro@hotmail.com \\ Guadalupe Fradejas Legido \\ Enfermera del complejo asistencial de Zamora .Servicio de endocrino.
}

Recepción Artículo: 06 diciembre 2021 Admisión Evaluación: 06 diciembre 2021 Informe Evaluador 1: 07 diciembre 2021 Informe Evaluador 2: 07 diciembre 2021 Aprobación Publicación: 07 diciembre 2021

\section{RESUMEN}

En la etapa de envejecimiento se producen cambios bio-psico-sociales que convierten a las población de edad avanzada en un colectivo altamente susceptible de presentar alteraciones en el estado nutricional que provoca cambios en la estructura del organismo, tales como la disminución de la masa celular, perdida de proteínas 0 alteraciones en relación a los líquidos intra y extracelular, esto repercute negativamente en el curso de enfermedades tanto en el desarrollo como en la recuperación.

Una valoración nutricional minuciosa es muy importante para poder hacer un abordaje nutricional apropiado, para ello nos podemos valer de múltiples escalas, valoraciones, y pruebas diagnósticas.

La utilización del análisis de impedancia bioeléctrica (BIA) se basa en la capacidad del cuerpo humano para conducir la corriente eléctrica. El análisis de la composición corporal consiste en el fraccionamiento de la masa corporal total en sus distintos componentes.

Mediante esta revisión bibliográfica desde de fuentes de mayor a menor evidencia, intentamos determinar si la realización de la técnica puede mejorar la calidad de vida de las personas mayores.

En virtud de los diferentes artículos estudiados, se observa variabilidad en cuanto los resultados obtenidos. Considerando la impedancia eléctrica como una herramienta útil no invasiva para el análisis de la composición corporal, si bien es verdad que se estima mayor error si el paciente presenta deshidratación o sobrecarga de líquidos.

Si lo valoramos en función de nuestro objetivo, nos lleva a la conclusión de que sería necesario una valoración exhaustiva, siendo beneficiosa en personas mayores con obesidad o con insuficiencia renal que son las patologías que han demostrado más eficacia para la técnica, sin embargo las personas con caquexia, con sobrecarga liquida por edemas o con signos de deshidratación podrían darnos error o no ser útil.

Palabras clave: impedancia eléctrica; composición corporal; agua corporal; anciano 


\section{LA UTILIDAD DE LA BIOIMPEDANCIA EN PERSONAS MAYORES}

\section{ABSTRACT}

The usefulness of bioimpedance in older people. In the aging stage bio-psycho-social changes occur that make the elderly population a highly susceptible group to present alterations in the nutritional status that causes changes in the structure of the body, such as decreased cell mass, loss of proteins or alterations in relation to intra and extracellular fluids, this has a negative impact on the course of diseases in both development and recovery.

A thorough nutritional assessment is very important to be able to make an appropriate nutritional approach, for this we can use multiple scales, assessments, and diagnostic tests.

The use of bioelectrical impedance analysis (BIA) is based on the ability of the human body to conduct electrical current. Body composition analysis consists of the fractionation of total body mass into its various components.

By means of this literature review from sources of greater to lesser evidence, we tried to determine whether the performance of the technique can improve the quality of life of the elderly.

By virtue of the different articles studied, variability is observed in terms of the results obtained. Considering electrical impedance as a useful non-invasive tool for the analysis of body composition, although it is true that a greater error is estimated if the patient presents dehydration or fluid overload.

If we evaluate it according to our objective, it leads us to the conclusion that an exhaustive evaluation would be necessary, being beneficial in elderly people with obesity or with renal insufficiency, which are the pathologies that have shown more efficiency for the technique, however, people with cachexia, with liquid overload due to edema or with signs of dehydration could give us error or not be useful.

Keywords: electric impedance; body composition; body water; aged

\section{ANTECEDENTES}

El proceso de envejecimiento de la población es unos de los factores que influyen en el aumento de prevalencia de desnutrición, los ancianos son el colectivo de riesgo por sus características biológicas, psicológicas y sociales. , la malnutrición por defecto es uno de los problemas más relevantes en la población anciana. Una valoración nutricional exhaustiva incluye como mínimo estos puntos:

1.Historia clínico-nutricional

2. Historia dietética

3.Valoración antropométrica.

4.Valoración bioquímica y datos del laboratorio.

5.Análisis de la composición corporal.

6.Valoración funcional.

Su finalidad es intentar identificar las causas específicas del riesgo nutricional y establecer el diagnóstico correcto para implementar el abordaje apropiado.

El uso de la impedancia para el análisis de la composición corporal se considera de gran precisión porque en un corto periodo de tiempo permite obtener datos para evaluar el estado de hidratación y nutrición en personas sanas y enfermas por diversas causas.

La técnica es un método no invasivo, que permite medir la composición corporal del organismo en unos minutos. Se basa en la capacidad de este para conducir la corriente eléctrica. Se define como la oposición de un conductor al flujo de la corriente eléctrica. Los parámetros dependen del contenido de agua y la conducción eléctrica. Es una herramienta usada para:

- Estimar la composición corporal en dos o tres compartimentos ( masa grasa, masa libre, masa extracelular).

- Monitorear el desgaste 0 deterioro celular.

- Evaluar el nivel y la distribución del agua corporal.

- Observar las fluctuaciones de los fluidos corporales. 
(a)

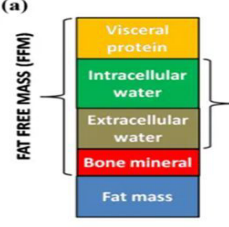

(b)

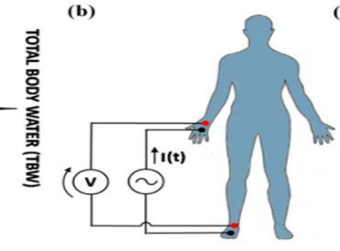

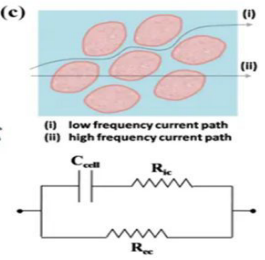

\section{OBJETIVOS}

Determinar si es necesaria una bioimpedancia para mejorar la valoración nutricional en personas mayores.

\section{MUESTRA}

Se seleccionan 5 artículos entre el año 2005-2017, por su utilidad para el objetivo que queremos realizar, entre los mismos incluimos un estudio específico con personas mayores más otros con otras patologías.

\section{METODOLOGIA}

Se realiza una revisión bibliográfica desde diferentes fuentes de datos de mayor evidencia hasta estudios relacionados con el uso y resultados de la bioimpedancia. Se consultan diferentes Meta buscadores, bases de datos y buscadores de evidencia: COHRANE,GUIA SALUD,MEDLININE ( PUBMED),CUIDEN,IBECS.

\section{RESULTADOS}

En virtud a los estudios seleccionados, se observa que para las personas mayores no es muy apta la bioimpedancia, aunque se trata de una técnica no invasiva nos aporta muy poca información. Si determina la composición de varios parámetros como M.L.G (masa libre de agua), MG (mas grasa) y masa celular que nos ayudarían a hacer una buena valoración y evolución de su estado nutricional, sin embargo en el estudio realizado a 69 personas mayores no se logra observar la utilidad y si se ven los errores cometidos por posibles deshidrataciones de los pacientes a estudio.

Por otro lado si se observa la utilidad de la técnica en pacientes de otro tipo como son las obesidades o los enfermos renales en tratamientos con diálisis.

\section{DISCUSION}

Para poder determinar si es útil o no la realización de la técnica, habría que realizar una valoración de la persona mayor, a pesar de que no es algo invasivo, lo lógico es ver la información que nos va aportar; para mejorar la calidad de vida del paciente, observando detenidamente el exceso o defecto de líquidos que es lo que nos proporcionaría el error, se rechazarían las personas con edemas y las caquexias o deshidrataciones.

Para el resto de personas mayores en función de su patología si podría beneficiarse, como podría ser el caso de obesidades o personas con insuficiencias renales.

\section{CONCLUSION}

En virtud de los diferentes artículos encontrados, seleccionados y analizados se observa variabilidad en cuanto los resultados obtenidos. Considerando la impedancia eléctrica como una herramienta útil no invasiva para el análisis de la composición corporal, pudiéndose estimar mayor error si el paciente presenta deshidratación o sobrecarga de líquidos.

A pesar de que existe un estudio de personas mayores que no logra demostrar la utilidad de la técnica, si realizamos el estudio general podemos concretar que si es muy útil para determinadas patologías que pueden presentar estas personas. 


\section{LA UTILIDAD DE LA BIOIMPEDANCIA EN PERSONAS MAYORES}

Por tanto se trata de hacer una buena valoración del estado del pacientes estimando el error que existe para determinar si sería practico o no realizar la bioimpedancia ,partiendo también de la base de que se trata de una técnica no invasiva y por tanto no contraindicada.

\section{REFERENCIAS BIBLIOGRÁFICAS}

1. Francisco José Berral de la Rosa, ${ }^{\star}$ Elizabeth Rodríguez Bies. Impedancia bioeléctrica y su aplicación en el ámbito hospitalario. Rev Hosp Jua Mex 2007; 74(2):104-112. Disponible en:

https://www.medigraphic.com/cgi-bin/new/resumen.cgi?IDARTICUL0=18663

2. Quesada LL, León RCC, Betancourt BJ, et al. Elementos teóricos y prácticos sobre la bioimpedancia eléctrica en salud. AMC. 2016;20(5):565-578.Disponible en:

https://www.medigraphic.com/cgibin/new/resumen.cgi?IDARTICUL0=68704.

3.Jose Maria Moran;Jesus Maria Lavado-Garcia;Juan Diego Pedrera Zamorano.Metodos de enfermería para la medición de la composición corporal.Rev. latino-am. Enfermagem vol19 July/aug.2011; Disponible: https://doi.org/10.1590/S0104-11692011000400024.

4. Hooper L, Abdelhamid A, Attreed NJ, Campbell WW, Channell AM, Chassagne P, Culp KR, Fletcher SJ, Fortes MB, Fuller N, Gaspar PM, Gilbert DJ, Heathcote AC, Kafri MW, Kajii F, Lindner G, Mack GW, Mentes JC, Merlani P, Needham RA, Olde Rikkert MGM, Perren A, Powers J, Ranson SC, Ritz P, Rowat AM, Sjöstrand F, Smith AC, Stookey JJD, Stotts NA, Thomas DR, Vivanti A, Wakefield BJ, Waldréus N, Walsh NP, Ward S, Potter JF, Hunter P. Clinical symptoms, signs and tests for identification of impending and current water loss dehydration in older people. Cochrane Database of Systematic Reviews 2015, Issue 4. Art. No.: CD009647. Disponible https: //doi: 10.1002/14651858.CD009647.

5. Camina-Martín MA, de Mateo-Silleras B, Malafarina V, Lopez-Mongil R, Niño-Martín V, López-Trigo JA, Redondo-Del-Río MP; Grupo de Nutrición de la Sociedad Española de Geriatría y Gerontología (SEGG). Valoración del estado nutricional en Geriatría: declaración de consenso del Grupo de Nutrición de la Sociedad Española de Geriatría y Gerontología. Rev Esp Geriatr Gerontol. 2016 Ene-Feb;51(1):52-7. Español. Disponible: doi: 10.1016/j.regg.2015.07.007. Epub 2015 Sep 19. PMID: 26388249. 